Article

\title{
Study on the Context-Aware Middleware for Ubiquitous Greenhouses Using Wireless Sensor Networks
}

\author{
Jeonghwang Hwang and Hyun Yoe*
}

School of Information and Communication Engineering, Sunchon National University, Maegok-dong, Suncheon-si, Jeollanam-do 540-742, Korea; E-Mail: jhwang@sunchon.ac.kr

* Author to whom correspondence should be addressed; E-Mail: yhyun@ sunchon.ac.kr; Tel.: +82-61-750-3591; Fax: +82-61-750-3590.

Received: 20 February 2011; in revised form: 2 April 2011 / Accepted: 10 April 2011 / Published: 27 April 2011

\begin{abstract}
Wireless Sensor Network (WSN) technology is one of the important technologies to implement the ubiquitous society, and it could increase productivity of agricultural and livestock products, and secure transparency of distribution channels if such a WSN technology were successfully applied to the agricultural sector. Middleware, which can connect WSN hardware, applications, and enterprise systems, is required to construct ubiquitous agriculture environment combining WSN technology with agricultural sector applications, but there have been insufficient studies in the field of WSN middleware in the agricultural environment, compared to other industries. This paper proposes a context-aware middleware to efficiently process data collected from ubiquitous greenhouses by applying WSN technology and used to implement combined services through organic connectivity of data. The proposed middleware abstracts heterogeneous sensor nodes to integrate different forms of data, and provides intelligent context-aware, event service, and filtering functions to maximize operability and scalability of the middleware. To evaluate the performance of the middleware, an integrated management system for ubiquitous greenhouses was implemented by applying the proposed middleware to an existing greenhouse, and it was tested by measuring the level of load through CPU usage and the response time for users' requests when the system is working.
\end{abstract}

Keywords: WSN; ubiquitous society; agriculture; context-aware; middleware; greenhouse 


\section{Introduction}

Wireless Sensor Network (WSN) technology is one of the important technologies for implementing a ubiquitous society, and it is applied into various fields such as distribution, logistics, construction, transportation, agriculture, defense, medicine, etc. [1], and in particular, it can be applied to the agricultural environment, production management, distribution, etc. to increase the productivity of agricultural and livestock products and secure transparency of distribution channels in the agricultural sector $[2,3]$.

Ubiquitous agriculture ( $\mathrm{u}$-Agriculture) is defined as a technology that increases agricultural added value and productivity by combining ubiquitous technologies such as WSNs with agricultural sector applications, and recently, various pilot projects and research projects involving the design and implementation of monitoring systems combining WSN technologies have been tried in various of agricultural and livestock production environments for product management and distribution in applications such as greenhouses and livestock barns, etc. [4-8].

In order to easily construct such a u-Agriculture environment, middleware is required to connect WSN hardware, applications, and enterprise systems $[9,10]$. It is said that middleware is a technology to filter a large volume of data collected from many heterogeneous WSNs, process event data, and then convert it into meaningful information, and furthermore, to transmit and process more efficiently a large number of contexts and data produced in a ubiquitous environment [11-16].

Recently, even though WSN middleware has been studied in various fields, there have been insufficient studies on middleware focused on application services in an agricultural environment where compared to other industries, IT technology has been inadequately applied [17]. Therefore, this paper would like to propose a context-aware middleware to efficiently process data collected from greenhouses by applying WSN technologies and to implement combined services through organic data connectivities.

In greenhouses using WSN technologies, soil sensor and environmental sensor nodes are installed inside/outside the greenhouse in order to collect environmental information for monitoring the greenhouse crop growth, and these sensor nodes constitute a wireless sensor network to collect environmental and soil information in the greenhouse.

In order to provide various services to users by using wireless sensor networks composed of heterogeneous sensors, WSN middleware should be capable of converting collected sensor data into a common form, reducing server loads by using some data filtering function(s), and providing intelligent context-aware and event service functions.

The proposed context-aware middleware could maximize scalability and usability of the system by abstracting heterogeneous sensor nodes installed for collecting greenhouse environmental information, enabling data filtering, event processing and context-aware processing, and integrating different forms of data through this.

The proposed context-aware middleware was applied to a ubiquitous greenhouse integrated management system, and we could thus maximize scalability and operability of the system and improve the productivity of greenhouse crops and user convenience.

This paper is organized as follows: Section 2 describes related studies, Section 3 explains design of the proposed context-aware middleware, Section 4 implements the proposed middleware and evaluates 
its performance through applied examples, and finally, Section 5 concludes this paper by describing the conclusions and the future study topics.

\section{Related Research}

\subsection{Wireless Sensor Networks Middleware}

WSN middleware is a software layer that exists physically between hardware such as sensor nodes, gateways, etc. and applications, that supports the flexible integration of hardware and applications. In addition, it could be defined as a software to help provide services such as distributed computing environments, remote procedure calls, messaging to users, regardless of the hardware, operating system, network, etc. used. The recently studied WSN middleware can be divided according to its location into in-network schemes to be installed at sensor nodes, server-side schemes installed at servers, and hybrid schemes combining two schemes [10].

\subsubsection{In-Network Schemes}

Typical WSN in-network scheme middlewares, in which the middleware is installed at sensor nodes, are Mate developed at UC Berkeley, Impala developed at Princeton University, and Agilla developed at Washington University.

Mate is a virtual machine based middleware developed for sensor networks. It works on sensor nodes by installing TinyOS, is equipped with its own byte code interpreter, and supports a mechanism to distribute new codes through a contagion model. The high-level interface of Mate makes a complex program very simple, and it minimizes the resources required to send a new execution module to sensor nodes [18].

Impala was begun as part of the ZebraNet project at Princeton University. ZebraNet is a project to study the movement and breeding of animals such as zebras using sensor network technologies. Impala focuses on modularization, adaptability, and restoration of applications; software updated in Impala is delivered to each sensor node via wireless networks, and each node could carry out updates under the condition that the system is working. In addition, it improves performance of software systems, energy efficiency, stability, and provides dynamic applied adaptability for various parameters and device failures, etc. [19].

Agilla is a mobile agent-based middleware to allow codes and states of sensor nodes to move using WSNs. Agilla controls ensure the flexible propagation of sensor node state information. Agilla works on TinyOS, and carries out agent's function at each node. Agilla provides a neighbor list and double space resources at each node. The neighbor list that includes addresses of neighboring nodes is used to move and copy information of each node. The double space provides a decoupled-mode for communication between agents [20].

\subsubsection{Server-Side Schemes}

Typical server-side WSN middleware schemes, in which the middleware is installed on servers, are Cougar developed at Cornell University, SINA developed at the University of Delaware, and MiLAN developed at the University of Rochester. 
Cougar is a distributed data processing system for sensor networks studied by the database research team at Cornell University. Even though most sensor network applications recently studied employ a scheme in which a base node collects all the data and data is processed at the center, Cougar carries out all the data access and processing in a distributed form. Cougar uses declarative queries so that it also has a property that users are hidden from the physical properties of the networks. It was designed as a system that could adapt dynamically to network variation, has high flexibility and scalability, and has fault tolerance [21].

SINA considers sensor networks as a distributed database to access sensor network information using a query form. SINA includes not only a method to restrict re-transmission of similar information from sensor nodes distributed at geographically close locations but also a lower level mechanism to perform hierarchical grouping of sensors for efficient data fusion [22].

MiLAN is middleware for sensor networks that was developed at the University of Rochester for health management tasks at smart medical homes. It computes a measurement plan to minimize total energy consumption to satisfy the reliability demands of various types of medical data desired by users, activates the corresponding sensors according to the plan, and provides services to collect measured data and deliver it to users [23].

\subsubsection{Hybrid Schemes}

Hybrid scheme WSN middleware combines two of the schemes mentioned earlier; typical WSN middleware of this type are COSMOS developed at ETRI, DSWare developed at Virginia University, and TinyDB developed at UC Berkeley.

COSMOS, developed at ETRI, extracts core functions of middleware commonly required in various types of WSN application services, and brings about technology development and standardization for providing them as a standardized scheme. Major functions of COSMOS are supporting various types of queries involving massive simultaneous query processing for a large volume of sensor network environments, and supporting an abstraction function for heterogeneous sensor networks [24].

DSWare carries out data service functions by providing combined events defined by a certain pattern as a basic programming function, and has a feature of variously providing also real-time data service such as definition of deadlines for event reports and intervals for certain events, etc. [25].

TinyDB is middleware studied at UC Berkeley. It is a query system to obtain information from sensor networks driven on TinyOS. In other words, TinyDB collects data from motes installed in the environment to filter and arranges it to for sending to outside PCs. TinyDB considers sensor networks as a virtual distributed database, and supports SQL-like query language and SRT. In addition, TinyDB provides a simple sensor API for making PC applications that could extract data from sensor networks through queries, and a query generator in GUI form and a program to display results that also uses this API. To use TinyDB, TinyDB components based on TinyOS should be installed on each sensor node in the sensor network [26].

\subsection{Comparison with Other Middlewares}

Xiong and his colleagues have proposed an optimization of existing methods, called tuning adaptive margin failure detector (TAM FD), which significantly improves quality of service (QoS), especially 
in the aggressive range and when the network is unstable [14]. In addition, he and his colleagues have proposed a novel and efficient distributed flow control scheme for multirate multicast (MR-M), based on the well known Proportional Integral and Derivative (PID) controllers [15].

Zhou and his colleagues have developed context-aware middleware for multimedia services in heterogeneous networks. This context-aware middleware system facilitates diverse multimedia services in such heterogeneous network environments by combining an adaptive service provisioning middleware framework with a context-aware multimedia middleware framework [16]. In addition, he and and his colleagues have developed fully distributed scheduling schemes with the goal of minimizing the video distortion and achieving certain fairness [17].

In this paper, we have studied context-aware middleware applied to the particular environment of a WSN based greenhouse. The proposed middleware has a different system structure from existing middlewares, and uses an ontology model for representation of context because ontology could define some information between concept and relationship, and handle new contexts easily by using rule-based reasoning functions.

\section{Design of the Proposed Context-A ware Middleware for a Ubiquitous Greenhouse}

\subsection{Ubiquitous Greenhouse}

\subsubsection{System Architecture}

The ubiquitous greenhouse (u-Greenhouse) is a system in which ubiquitous technology is applied to a greenhouse to control the environmental monitoring and control facilities of the greenhouse. The $\mathrm{u}$-Greenhouse of this paper applies a WSN, which is a ubiquitous technology, as a basis system of the greenhouse [27].

In the u-Greenhouse of this paper, soil sensors and environmental sensors are installed inside/outside the greenhouse in order to collect environmental data relevant to the greenhouse's crop growth such as illuminance, temperature, humidity, $\mathrm{CO}_{2}$ level, etc. and soil information like soil humidity, soil temperature, etc., and these sensors together constitute a wireless sensor network to collect environmental and soil information from the greenhouse. In addition, CCTVs are installed inside/outside the greenhouse to collect real time image information to provide additional greenhouse and crop image information and preventing dangers such as burglary and fire.

Such collected environmental and image information is stored in a server via the gateway, provided to users in real time through various interfaces, and environmental control facilities in the greenhouse can be automatically or manually controlled to ensure an optimum growth environment for the cultivated crops based on the collected information. Figure 1 shows the system structure of the ubiquitous greenhouse. 
Figure 1. System structure of a ubiquitous greenhouse.

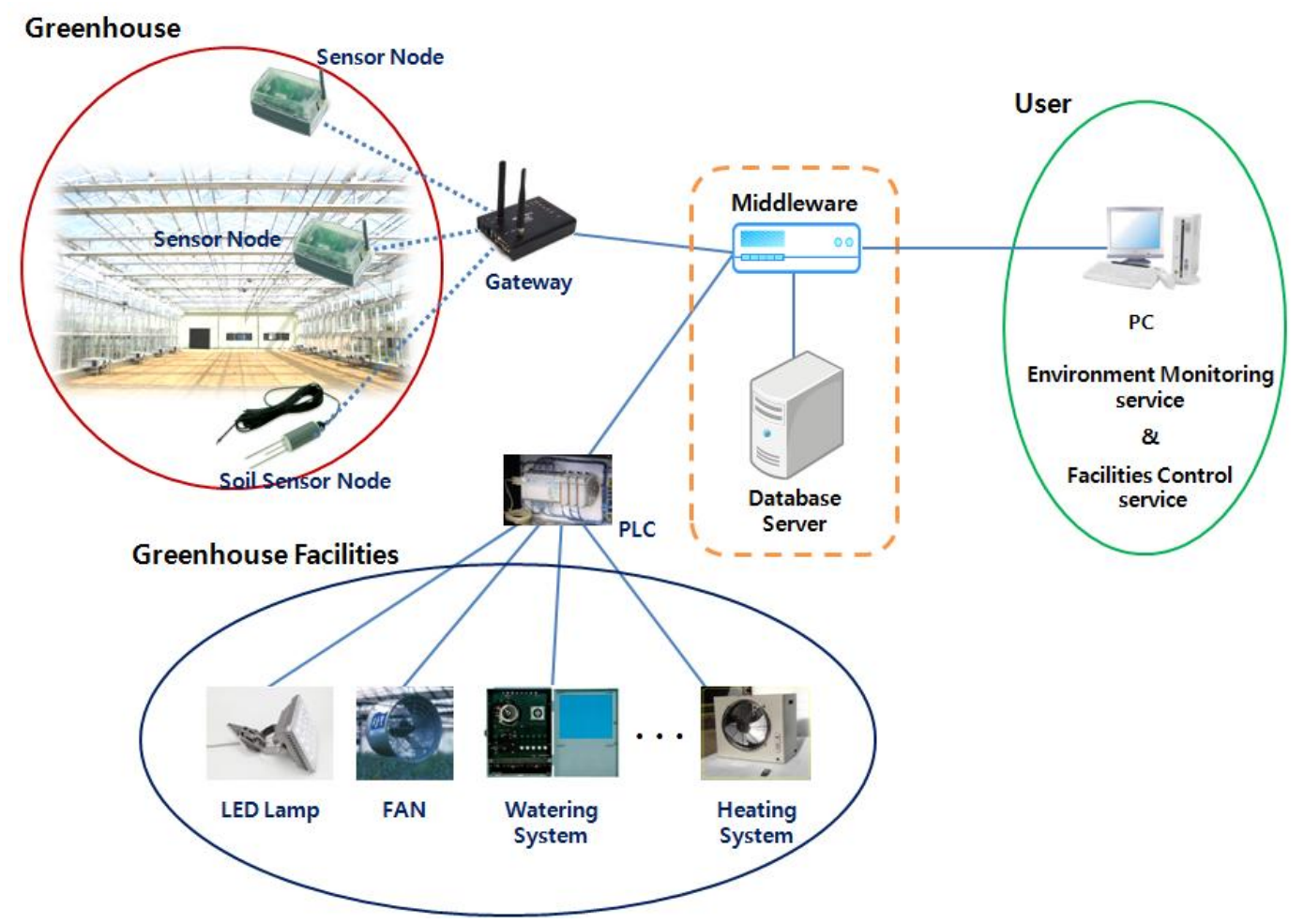

\subsubsection{System Components}

The u-Greenhouse has installed ZigbeX 2.0 sensor nodes from Hanback Electronics and developed sensor nodes inside/outside the greenhouse in order to measure environmental information such as temperature, humidity, illuminance etc. in the greenhouse.

Figure 2. ZigbeX 2.0 sensor node from Hanback Electronics.

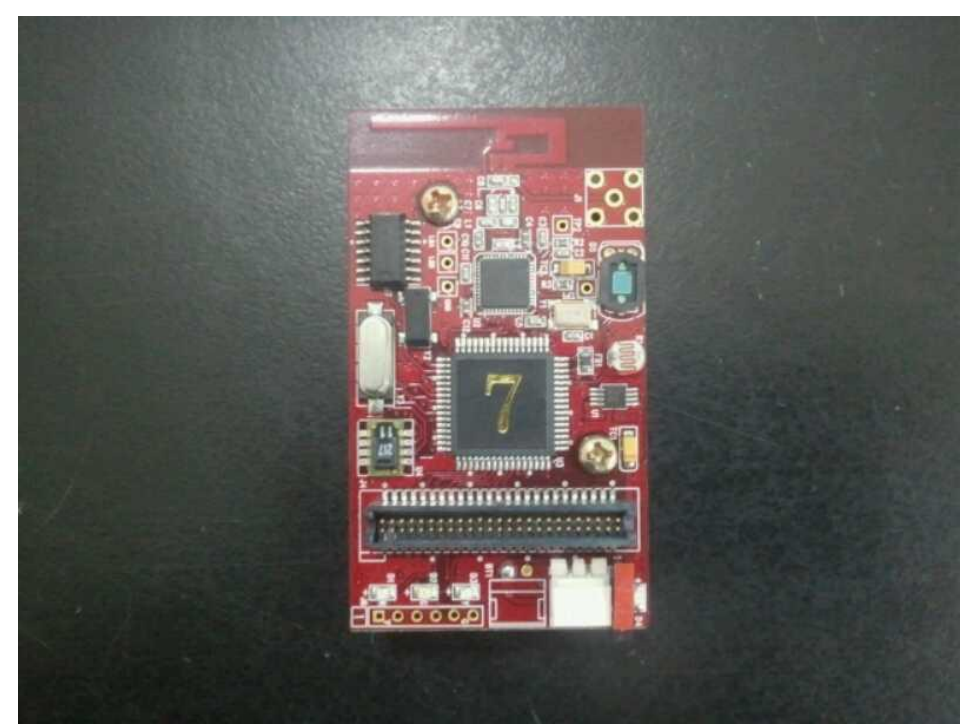

Figure 2 shows a ZigbeX 2.0 sensor node from Hanback Electronics. It is equipped with Atmel's ATmega128L with $128 \mathrm{~KB}$ internal programmable RISC architecture as a MCU, and Chipcon's CC2420 is used as a RF Transceiver. This ZigbeX unit outputs digital temperature/humidity data 
through a 14-bit ADC containing SHT11 temperature/humidity sensor as a multi-sensor device, and includes a Cds illuminance sensor with the maximum sensitivity at $540 \mathrm{~nm}$. For power it uses two rechargeable AA size batteries of between 2.7 3.6 VDC (1.2 rechargeable battery/1.5 Alkaline). The antenna for the RF Transmitter/Receiver is an F-type, which basically uses a PCB antenna with outdoor/indoor radius of $75 \mathrm{~m} \sim 100 \mathrm{~m} / 20 \mathrm{~m} \sim 30 \mathrm{~m}$, and a dipole antenna could be used as an option by users [28].

Figure 3 is a developed sensor node, which plays the role of receiving sensor data from the temperature/humidity sensor, processing data in the MSP430 MCU to send it to a relay node and a gateway via a CC2420 RF chip. In addition, the node is separated from the sensor in order to reduce the effects of any heat generated by the node on the sensor [29].

Figure 3. Environmental sensor node.

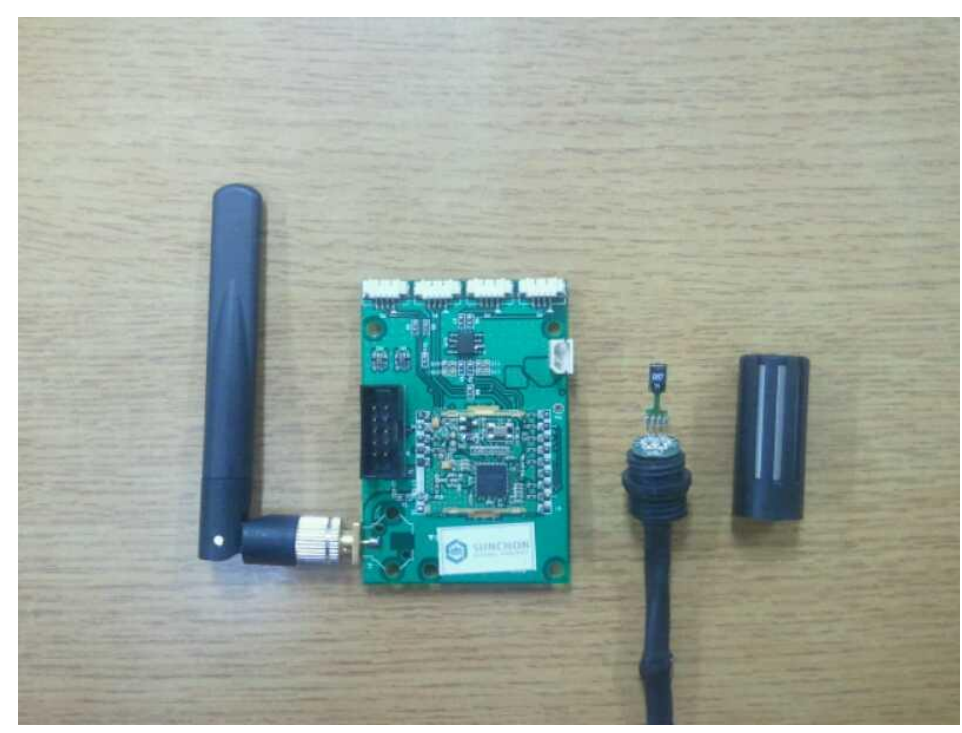

The MSP430 used in the sensor node is a 16-bit RISC, which has 48 Kbyte of program memory and 10 Kbyte RAM inside [30], so that it can process sensor data at high speed, and the CC2420 is a RF chip supporting ZigBee, which in turn supports 2,400 2,483.5 MHz band, works as a DDDS scheme, and supports O-QPSK modulation scheme and has a $250 \mathrm{Kbps}$ data rate so that low-power real-time wireless communication is possible [31].

SHT71 is used as an integrated temperature/humidity sensor. Operating power $(2.4 \mathrm{~V} \sim 5.5 \mathrm{~V})$ is relatively low and power consumption is also low, with an average value of $28 \mu \mathrm{A}$. It has a compensating memory, 14-bit A/D converter, digital 2-wire interface inside, and it can measure temperatures from -40 to $120{ }^{\circ} \mathrm{C}$ and has an accuracy error of $\pm 0.5{ }^{\circ} \mathrm{C}$. In addition, humidity is measured between 0 and $100 \%$, with an accuracy error of $3.5 \% .3 .3 \mathrm{~V}$ of operating voltage is connected to the sensor node, and digital 2-wire is connected with circuits of MSP430 to process the greenhouse temperature and humidity information [32].

Figure 4 shows a sensor node used to measure soil information such as soil temperature, soil humidity, etc. in the greenhouse. The soil sensor can store data by date using a downloading program without a data logger when connected with a computer via a relay. It can measure soil moisture from 0 
to $99.9 \%$, and the error rate is $\pm 3 \%$. Soil temperature can be measured from 0 to $60{ }^{\circ} \mathrm{C}$, and the error rate is $\pm 0.5^{\circ} \mathrm{C}$, so that its performance is excellent.

Figure 4. Soil sensor node.

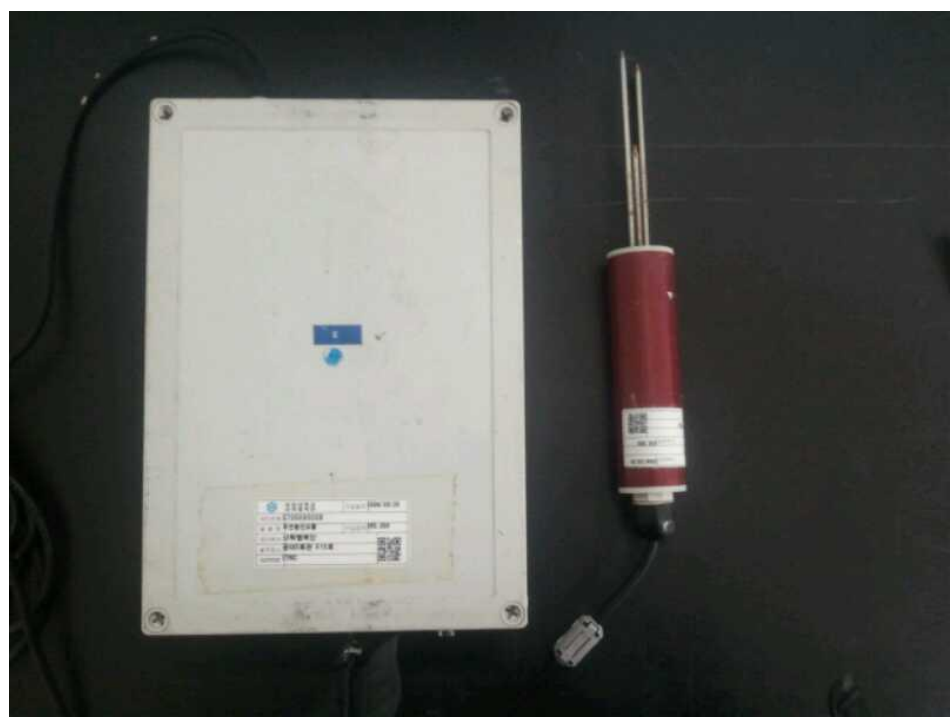

To control greenhouse environment parameters influencing crop growth such as illuminance, temperature, humidity, $\mathrm{CO}_{2}$, etc. based on information collected from the environmental sensors and the soil sensor mentioned above, environment control facilities such as a ventilation and heating system, heat insulation system for reducing energy, shading curtain system following external brightness, circulating fan system regulating air flow in the facility, temperature control system of hot water/heating water, and artificial light source control system following external brightness and biometric information etc. are constructed in the greenhouse, and each environment control facility is controlled through a PLC. Figure 5 shows the environment control facilities and the PLC installed in the greenhouse.

Figure 5. Environment control facilities and PLC.

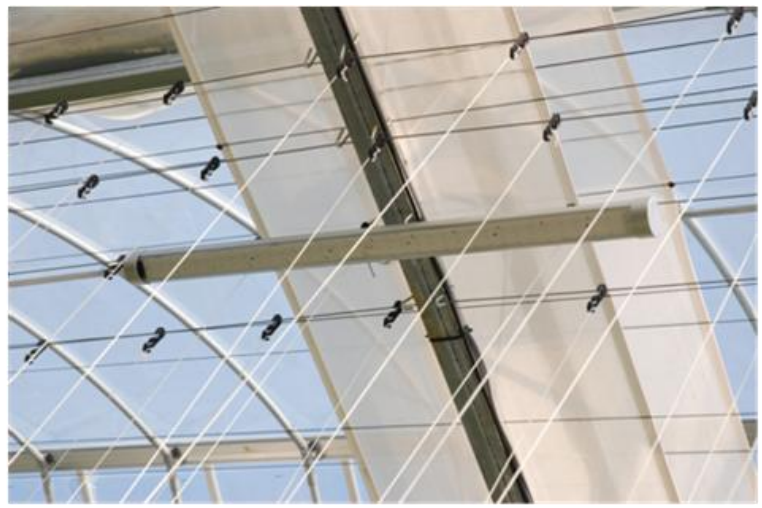

(a) LED L amp

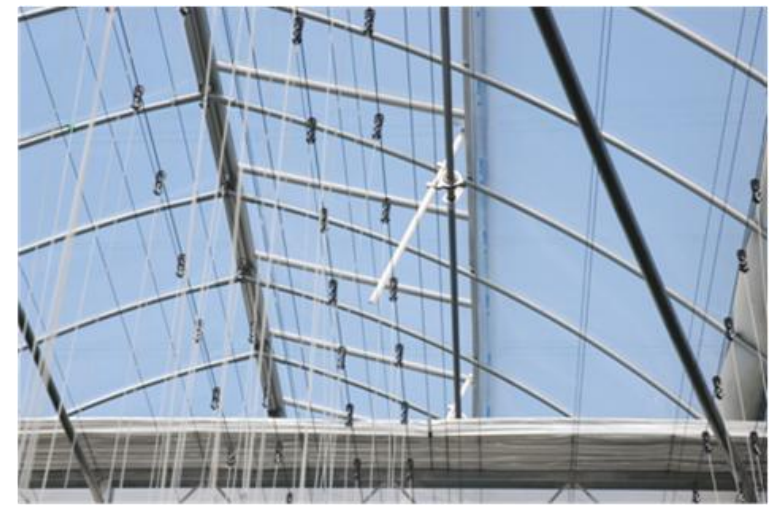

(b) Window 
Figure 5. Cont.

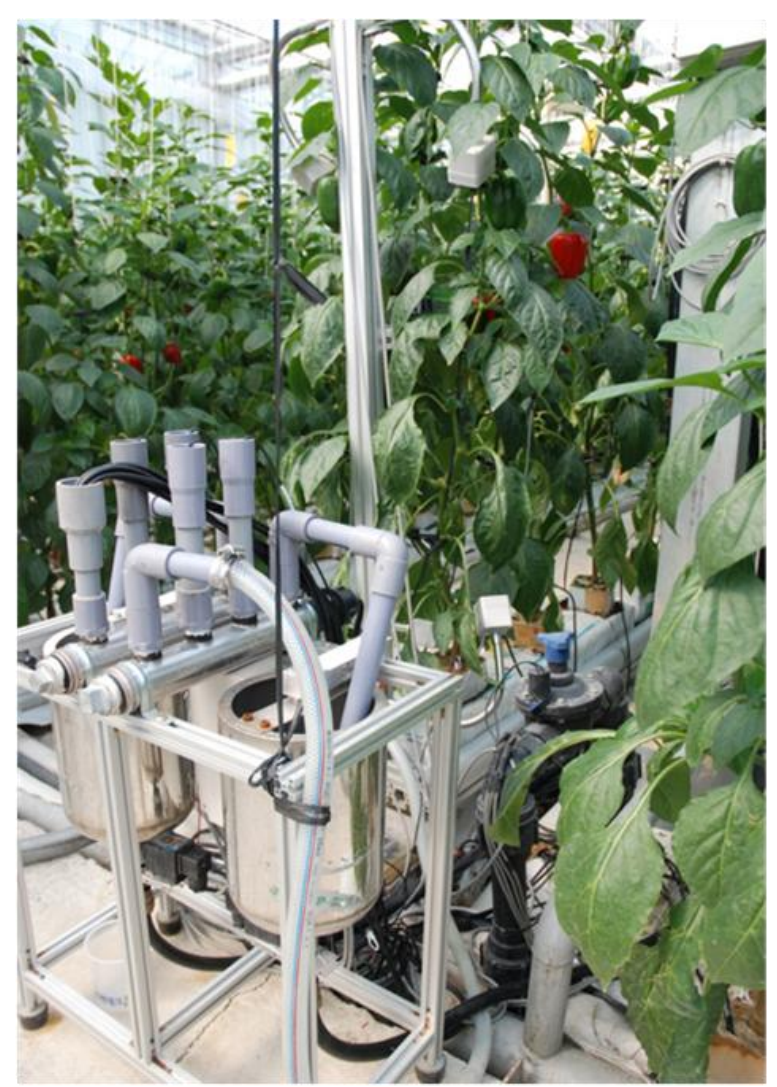

(c) Watering System

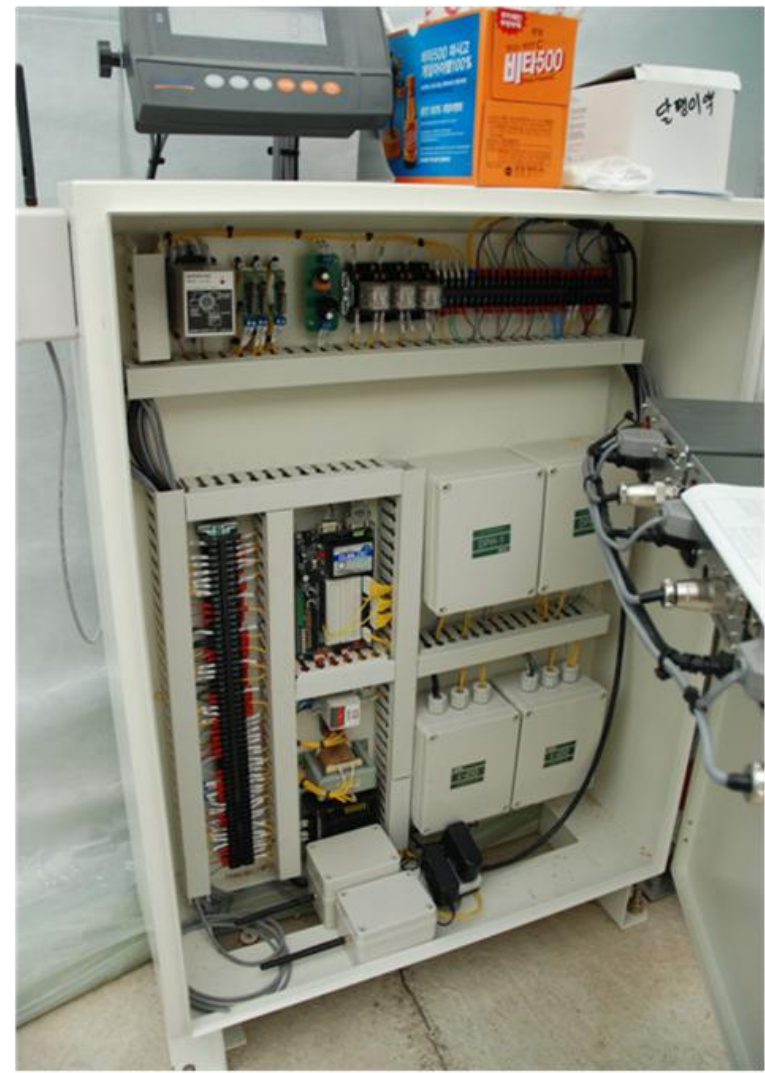

(d) PLC

\subsection{Design of the Proposed Context-Aware Middleware}

The context aware u-Greenhouse middleware is designed aimed at a system to smoothly interconnect two layers between the wireless sensor network basically composed of heterogeneous sensors and the system to provide various services to users, and to process data to effectively provide intelligent service functions that could recognize contexts based on sensor data.

\subsubsection{Requirements Analysis}

Requirements for the middleware analyzed in this paper could be divided into three parts: sensor network interface, data process, and application service interface, according to the location of each function. The sensor interface carries out data abstracting functions, and the data process provides data filtering, various query processing functions for sensor data, and real-time management functions for sensor information. In addition, the application service interface supports connections with the outside through query processing, context aware services, and event services.

The data abstracting function solves the dependency of sensor nodes by recognizing sensors and converting collected sensor data into a common form after registering heterogeneous sensor nodes, which are ported on each other's platform, to the middleware in advance.

The data filtering function is to avoid storing duplicated data to reduce the server's load for data sent in real time, and the context aware service is a function that specifies numerical values for certain data and decides contexts intelligently, to autonomically control greenhouse facilities appropriately for 
an optimum crop growth environment in the greenhouse. The event service prevents unexpected accidents by sending an urgent message to producers or managers if greenhouse environmental data, which is collected from sensors in real time, corresponds to a some configured event value.

\subsubsection{Middleware Scenario}

Figure 6 represents the functional demand analysis of the middleware system as a use case diagram. Producers or managers use the two context aware service and event processing service functions, and can control the greenhouse control facilities automatically or manually.

Figure 6. Use case diagram of the ubiquitous greenhouse system.

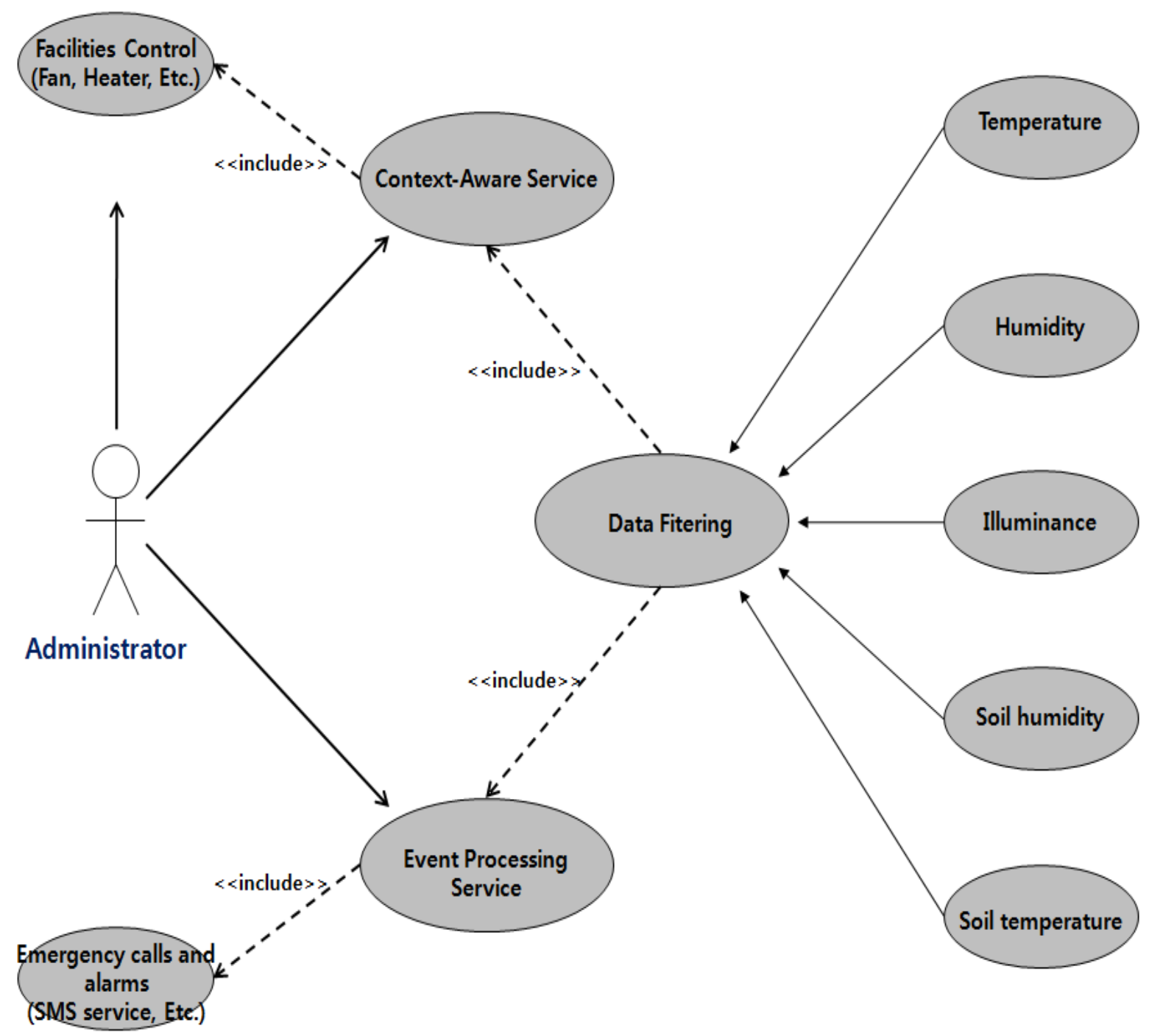

Figure 7 shows the process whereby the context aware service is carried out. If sensor data is entered from sensors, this data is sent to the middleware to perform the analysis and filtering process, and the autonomous context aware service is performed if it corresponds to a configured value of the context aware service. 
Figure 7. Sequence diagram of context aware service.

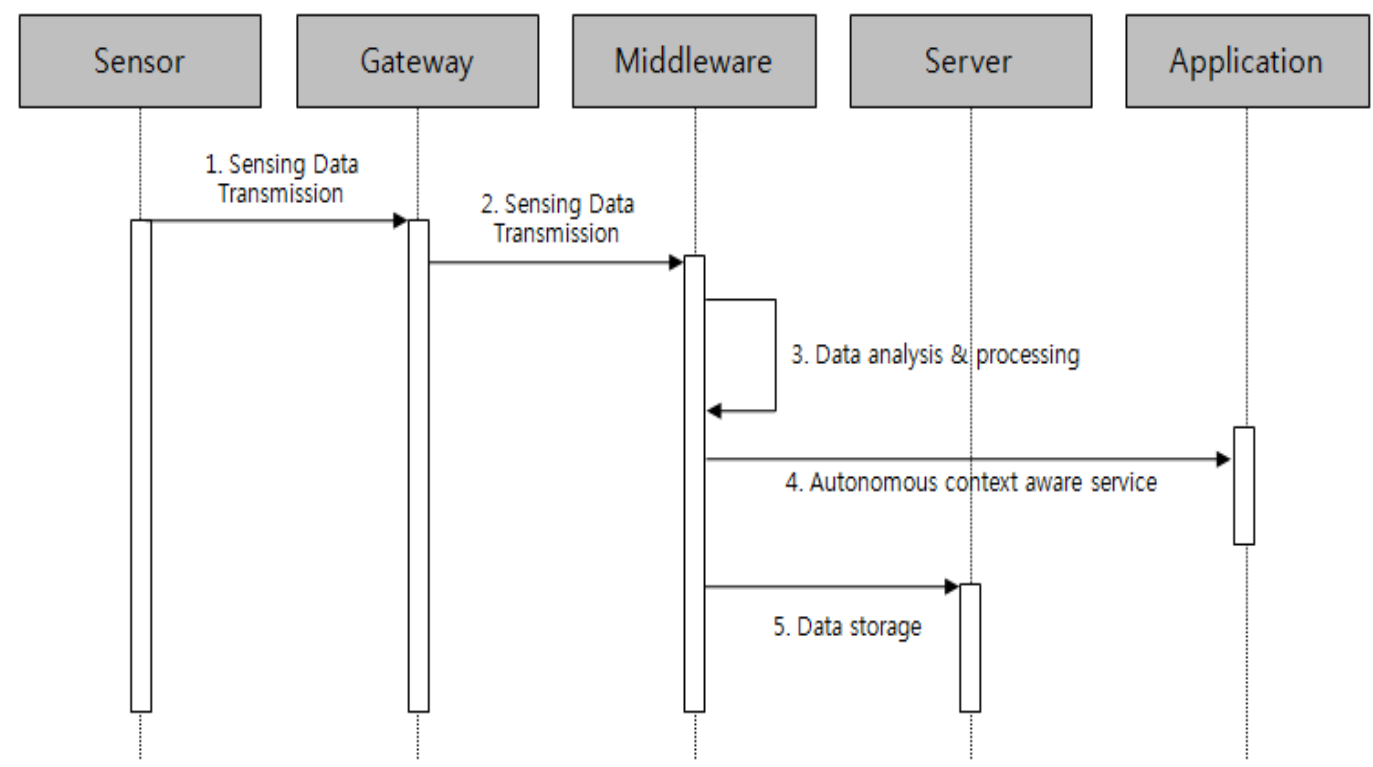

Figure 8 shows the process whereby the event service is carried out. Like the context aware service, it analyzes and processes data, which is sent to the middleware, to perform the event service if the data corresponds to a configured value of the event service.

Figure 8. Sequence diagram of event service.

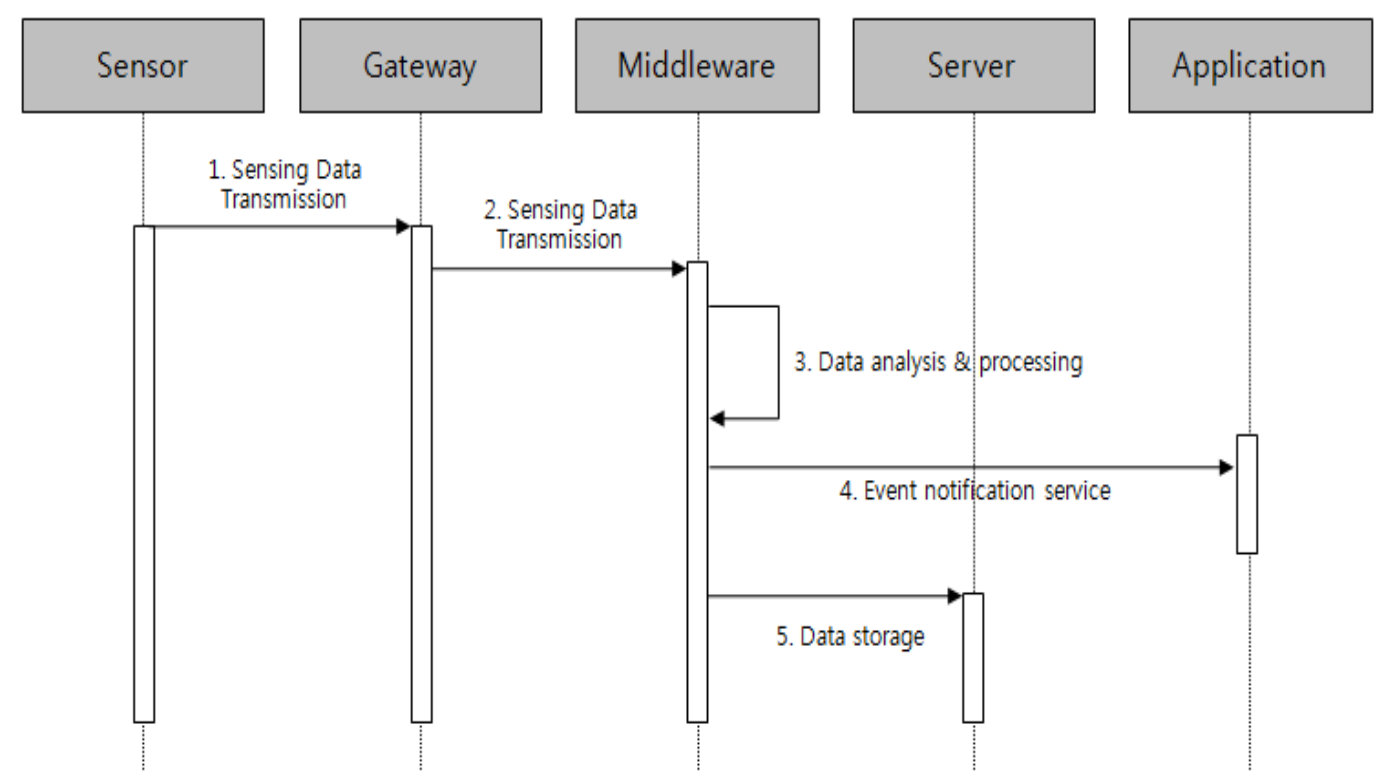

Figure 9 represents a process that requests greenhouse data and image information stored in the database (DB), and the requested data is received. If the application manager accesses the middleware to request greenhouse information, the middleware requests the greenhouse information stored in the DB to be sent, and sends the received information to the application to make the greenhouse information monitoring possible. 
Figure 9. Sequence diagram of the greenhouse information search service.

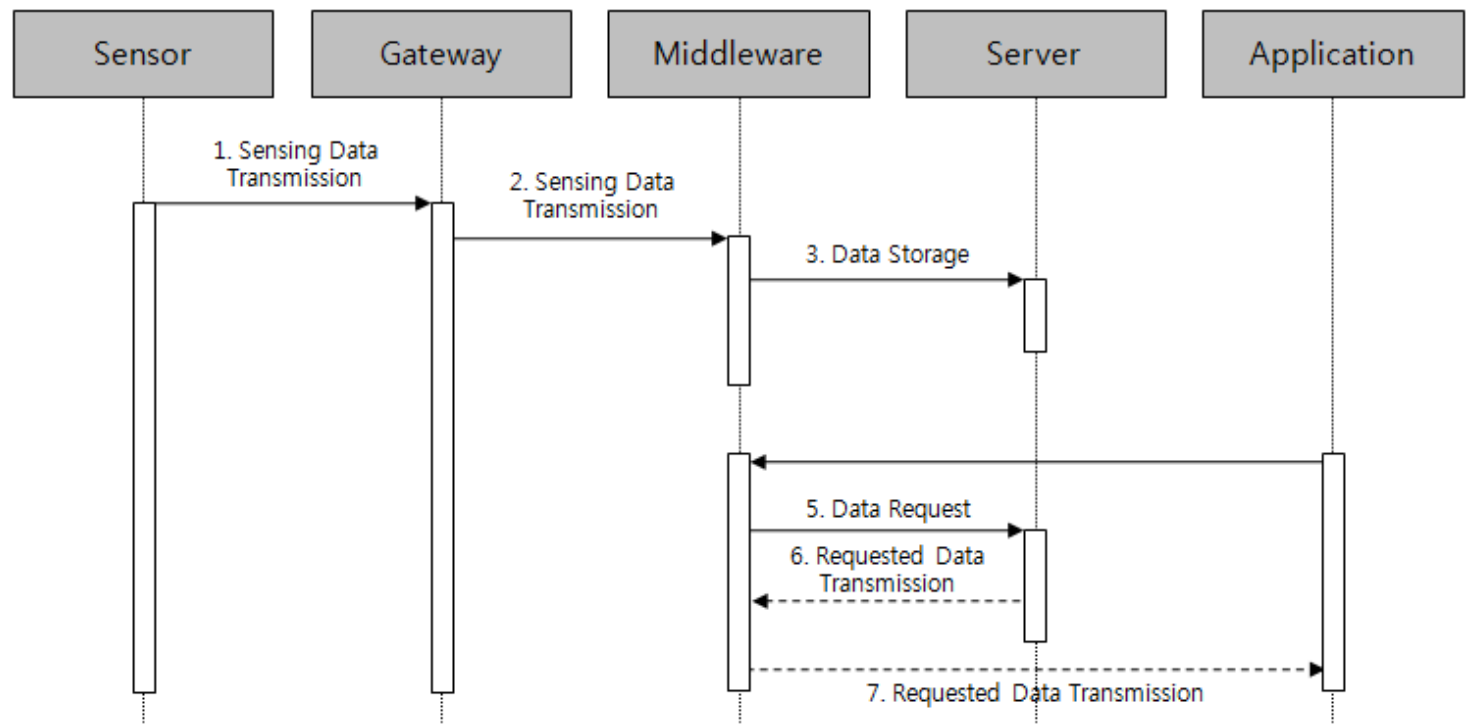

\subsubsection{Middleware Design}

The context aware middleware for $\mathrm{u}$-Greenhouses is composed of a sensor network interface layer, data process layer, and application service layer, and Figure 10 shows an hierarchical diagram of the proposed middleware.

Figure 10. Hierarchical diagram of the proposed middleware.

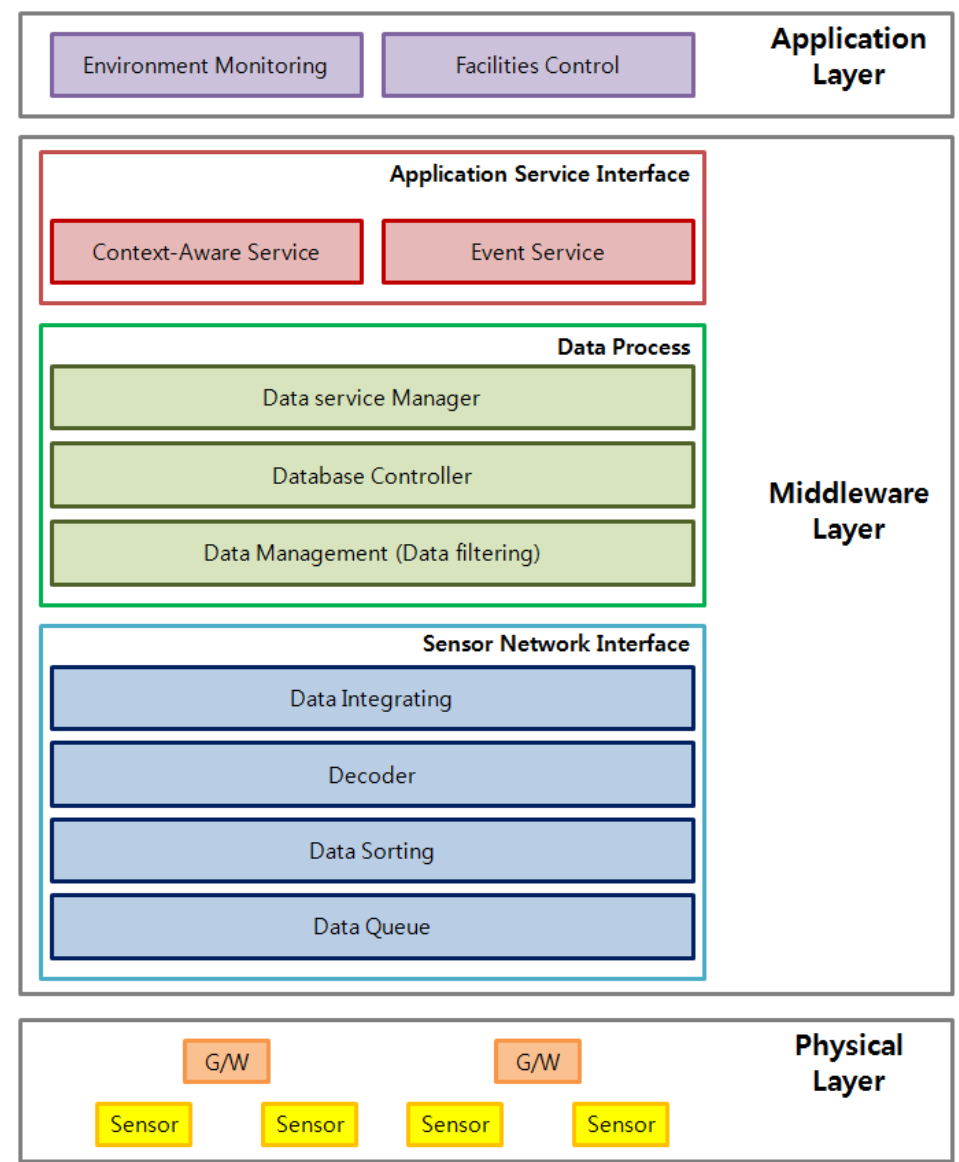


The sensor network interface layer is aimed at providing common interface functions to various multiple heterogeneous sensor networks, continuous monitoring and control functions for the states of various sensor networks.

The data process layer plays the role of providing various query processing functions for sensor data collected from the WSN infra and real-time management functions of sensor information. In addition, a sensor data management component is placed in the data processing layer to filter sensing data and support various forms of queries.

The application service interface layer, which is the top layer of middleware, plays the role of providing context aware and event processing services, and supports connections with the outside through query processing.

Figure 11 represents the structure and data flow chart of the proposed middleware. Figure 11 shows functions for each module of the middleware and a structural flow with the sensors, gateway, and monitoring system.

Figure 11. Structure and data flow chart of the proposed middleware.

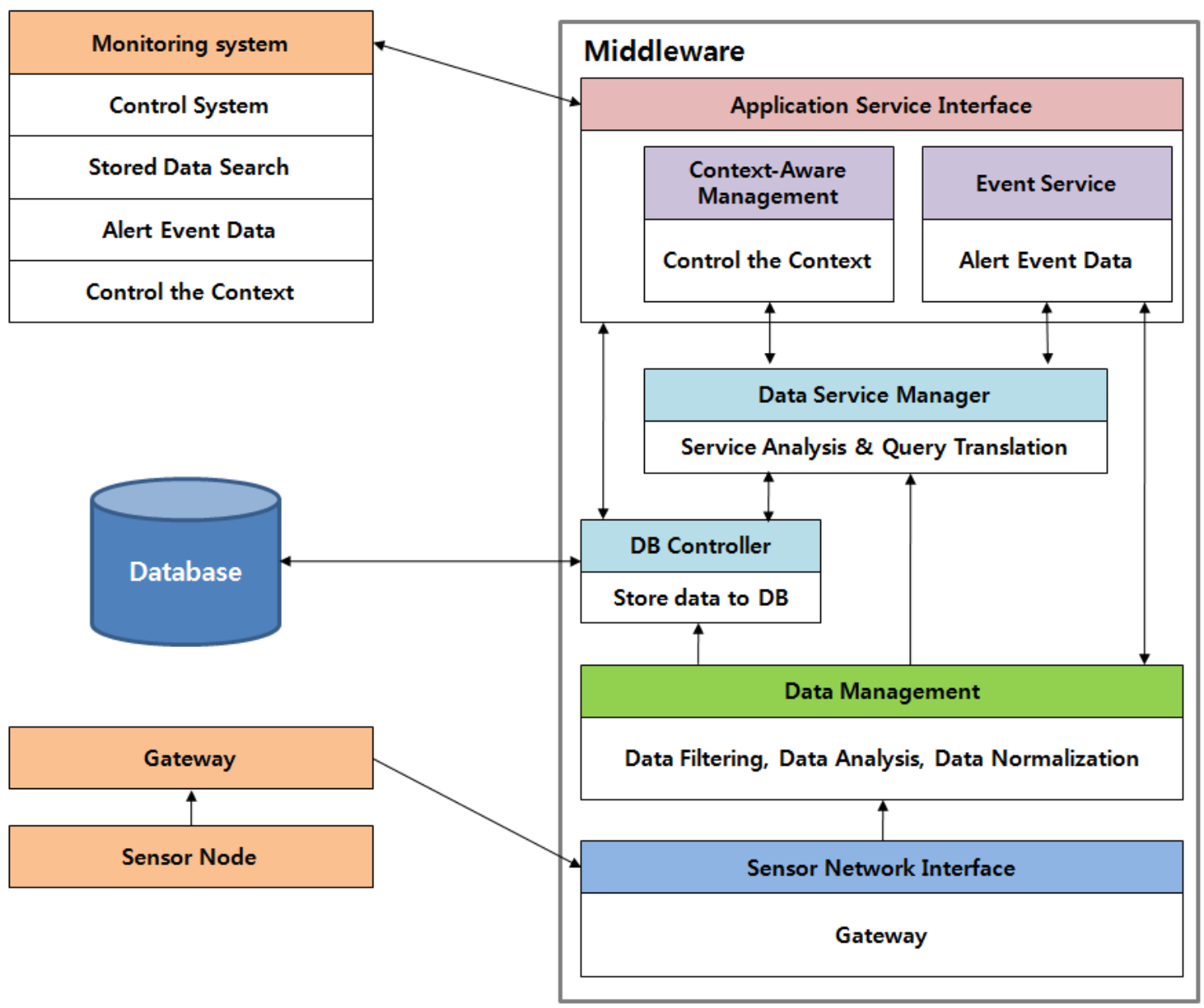

The sensor network interface stores the different data forms of the heterogeneous sensor nodes in advance to make sure all the data ported in the heterogeneous sensor network environment can be recognized. The most ideal situation is to have an integrated transducer to convert into a common form all the data obtained from any heterogeneous sensor, however, since a standard for sensor node's data 
forms is not established yet, this paper describes the conversion of two forms of data in the narrow concept into a specified common form.

The data filtering does not store data delivered from the sensor network interface in the DB if the data is a duplicate, or stores data only when the data is changed. In addition, it makes context awareness possible by comparing with the data value configured in advance by the application to notify the context aware management module if it is identical or exceeds some threshold valued. Figure 12 shows the structure and working process of the context aware management module.

Figure 12. Structure and working process of the context aware management module

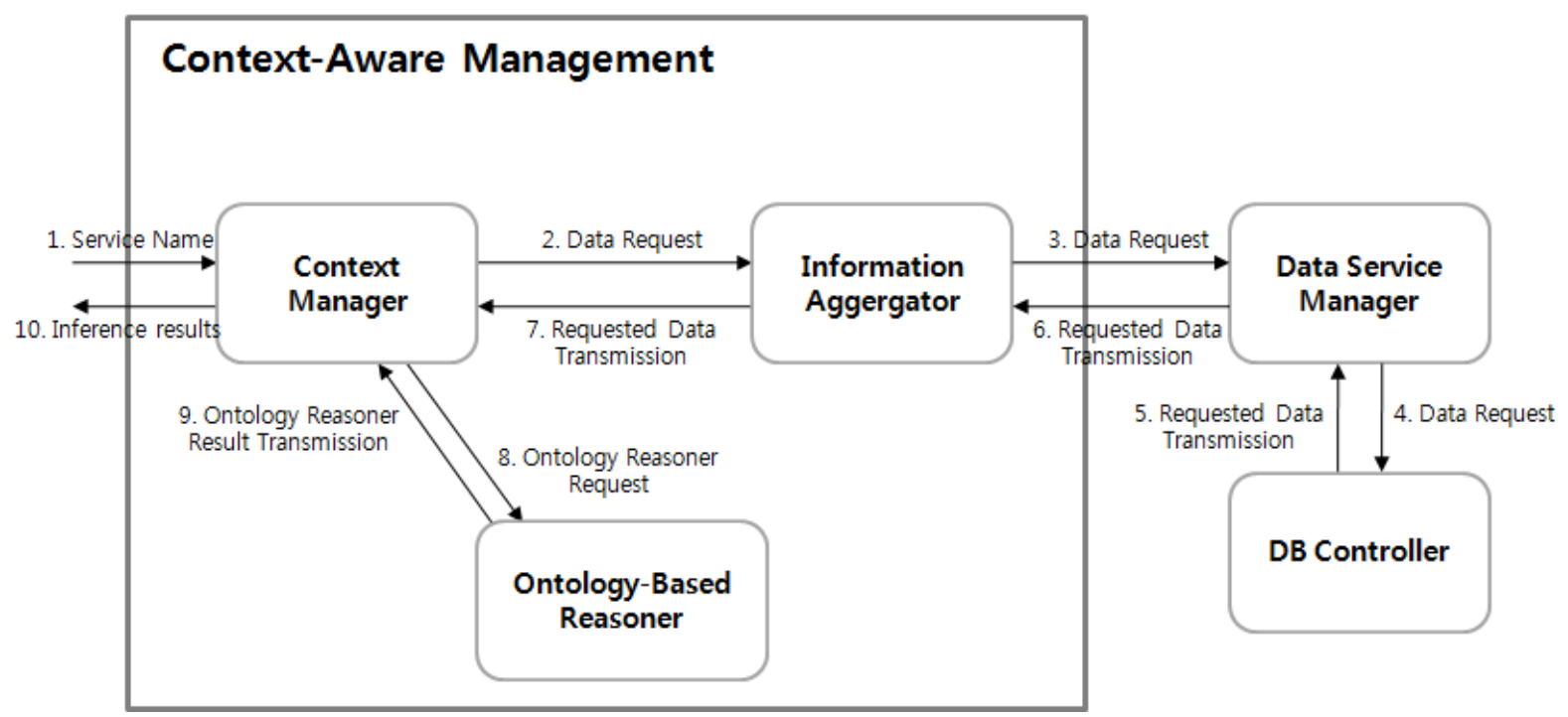

In addition, if the data entered has the same value as the level requested by an application, it notifies the event processing service module to make the event service possible. The usual sensing data not corresponding to such a process is delivered to the DB controller for storage in the DB.

The context-aware management module carries out the autonomous context-aware service to control the environmental control facilities in the greenhouse such as maintaining an optimum growth environment for the crops in the greenhouse depending on the data values based on data delivered from the data filtering. Action_Value and Data_Value are stored in the DB after the context-aware service.

The event service provides event services demanded by applications based on filtered data. It is a service to perform events specified by an application when receiving data corresponding to certain range of data values requested in advance. External intrusions and notifications of dangerous situations etc. correspond to this scenario. Action_Value and Data_Value are stored in the DB after the event service. The DB controller plays the role of sensing values delivered from the data filtering in the DB.

The application service interface plays the role of delivering and configuring a range of context aware services and event services configured by an application. And, it could search and store data in the database for items demanded by an application. In addition, it supports various queries to ensure flexible connections between applications and hardware are achieved. 


\section{Implementation of the Proposed Context-Aware Middleware for an Ubiquitous Greenhouse}

\subsection{Implementation Environment}

The environment to implement the context-aware u-Greenhouse middleware is divided into hardware and software environments, and the details are as follows: the hardware environment to develop the proposed context-aware middleware is the server-side PC development environment, and the details are shown in Table 1.

Table 1. Hardware Environment.

\begin{tabular}{|c|c|c|}
\cline { 2 - 3 } \multicolumn{1}{c|}{} & Type & Details \\
\hline \multirow{3}{*}{ Server PC Environment } & CPU & Intel Xeon 3.2 Ghz \\
& RAM & $1 \mathrm{~GB}$ \\
& OS & Microsoft Windows XP \\
\hline
\end{tabular}

In the hardware environment, the PC development environment corresponding to a server is implemented as the general PC environment, and the sensor node environment in charge of sensing is constructed as the environment suitable to use Zigbee sensors.

The software environment to develop the proposed context-aware middleware is as outlined in Table 2. In the software environment, the PC development environment uses a Microsoft Windows series operating system, Java and C\# as programming language the and database is implemented with MySQL. The sensor node environment responsible for sensing is constructed as a Linux environment in the Windows environment with Cygwin, and it uses TinyOS as the operating system to produce the sensor program with NesC.

Table 2. Software Environment.

\begin{tabular}{|c|c|c|}
\cline { 2 - 3 } \multicolumn{1}{c|}{} & Type & Details \\
\hline \multirow{3}{*}{ PC Development } & OS & Microsoft Windows XP \\
Environment & Programming & JAVA (JDK 6), C\# \\
& Languages & MySQL 5.0 \\
\hline & RDBMS & TinyOS 1.0 \\
Sensor Node & OS & Cygwin \\
Environment & Linux Environment & NesC \\
& Sensor Programming & JDK 1.4.1 \\
\hline
\end{tabular}

\subsection{Implementation of the Proposed Context-Aware Middleware}

\subsubsection{Middleware Algorithms}

Figure 13 is the algorithm that processes data which is delivered from the gateway in the implemented middleware, to store it in the DB. 
Figure 13. Middleware Algorithm.

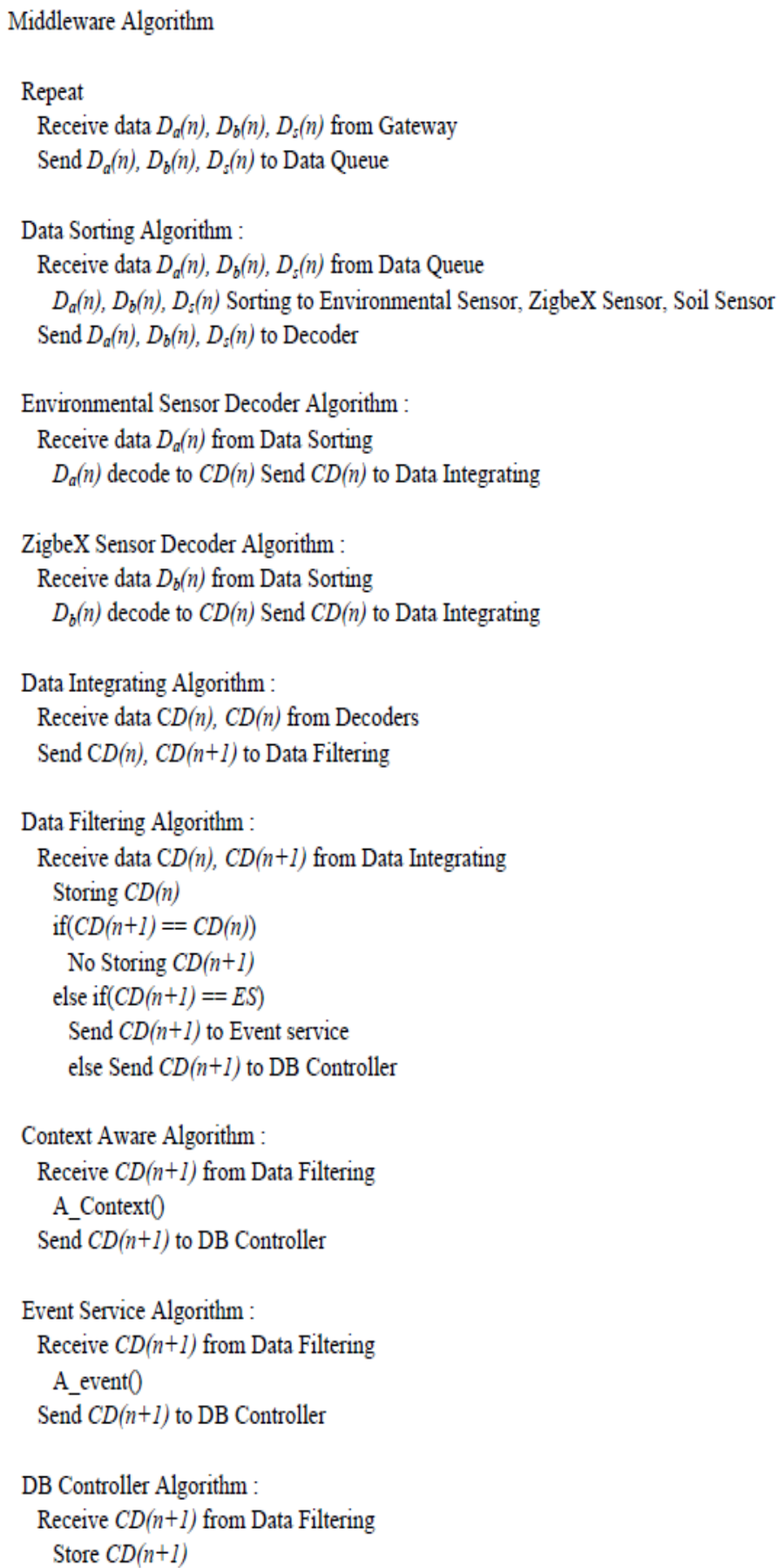

Data of $D a(n), D b(n)$, and $D s(n)$ obtained from the heterogeneous sensor networks are divided into each form in the data sorting module to send to each decoder. Each decoder converts $D a(n), D b(n)$, and $D s(n)$ into the common form $C D(n)$, and sends to the data integrating module to integrate it as $C D(n)$ and $C D(n+1)$ in order.

Data integrated as the common form is not sent to the DB controller module and dropped if it is duplicated data by comparing $C D(n)$ and $C D(n+1)$ continuously entered through the data filtering module. 
In addition, when data identical to the data range configured in advance by the context-aware and event service module is sent, it goes to the context-aware and event service, respectively, to make them perform the corresponding action. The remaining values are sent to the DB controller module to store in the DB.

\subsubsection{Sensor Data Structure and Data Conversion}

The data obtained from heterogeneous sensor networks with sensors measuring illuminance, temperature, humidity, EC, $\mathrm{pH}$, soil humidity, etc. is converted into a common form for use. This is possible by converting the data structure in the packet units into the specified common form.

Figure 14 shows the structure of the message (TOS_Msg msg[],) which sends data defined already for abstraction converting into the common form, and data structure int8_t data[TOSH_DATA_LENGTH] containing the corresponding data.

Figure 14. Sending message structure and data conversion.

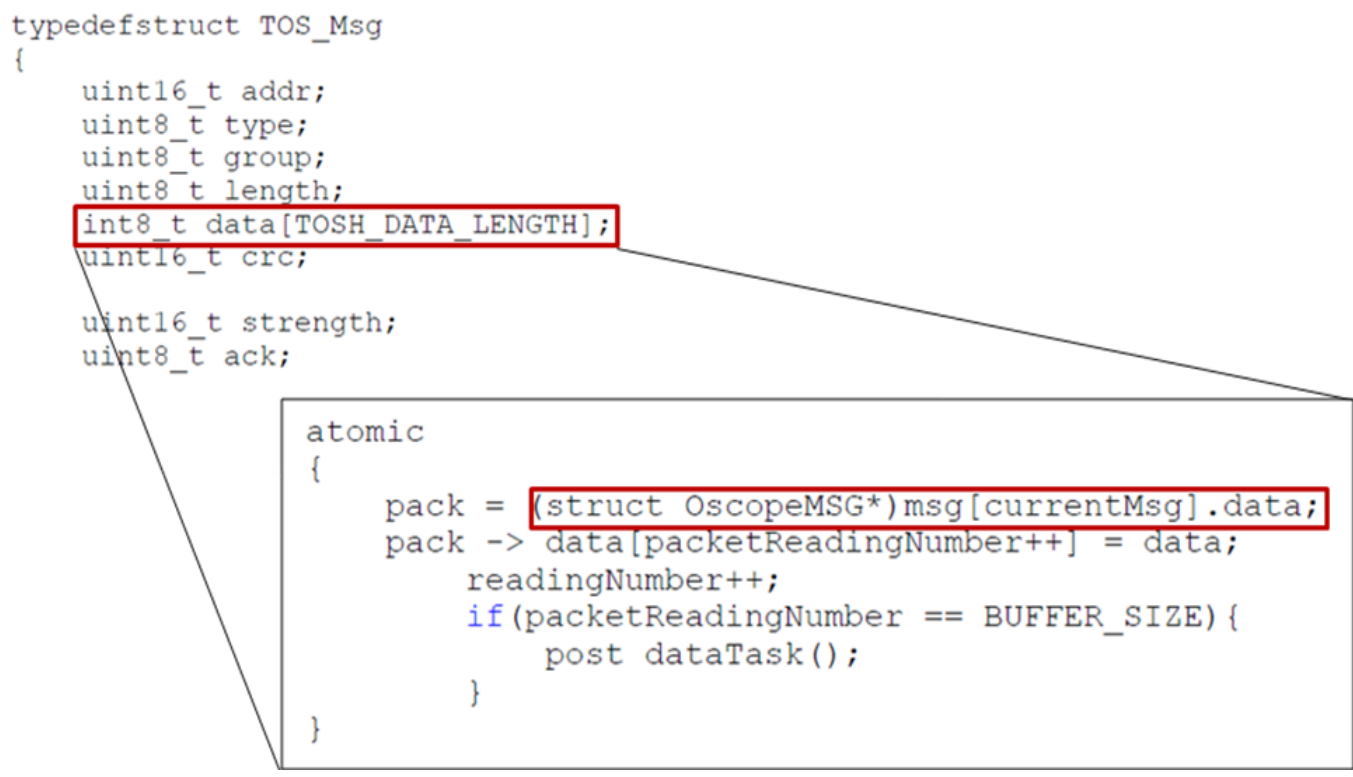

\subsubsection{Implementation of Ontology for Context-Aware Service}

To provide context-aware service, an ontology is constructed as shown in Figure 15; the ontology design uses Protege [33]. The ontology of this paper has six higher classes. Network represents information of sensor networks, and Sensor, Node, Location, and Context represent sensor, node, location, and context information, respectively, and Service represents services that users could be provided in the u-Greenhouse. Such information is written as OWL [34] documents and used with JENA [35]. 
Figure 15. Class hierarchies of ontology.

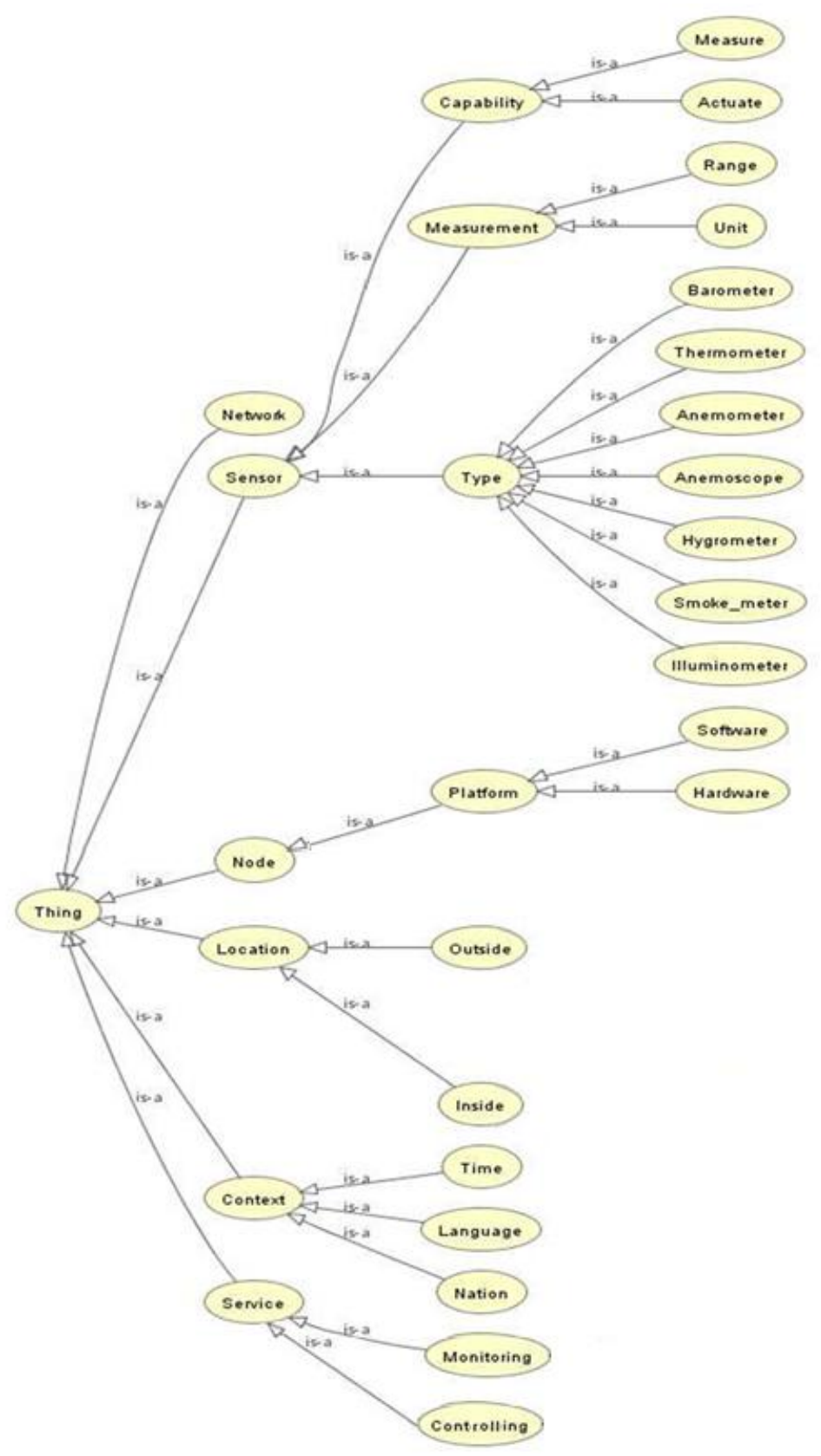

\subsection{Application of the Proposed Middleware}

The proposed middleware in this paper was applied to an existing $\mathrm{u}$-Greenhouse to implement the $\mathrm{u}$-Greenhouse integrated management system. Figure 16 shows the UI of u-Greenhouse integrated management system applying the proposed middleware. The system UI displays information such as illuminance, temperature, humidity, $\mathrm{CO}_{2}$, etc. collected from the environmental and soil sensors installed in the greenhouse as a text form, enabling real-time image information of the greenhouse and also controlling the CCTV, and allowing notification of the working state of the greenhouse environmental control facilities and their control. In addition, it was implemented so as to allow managers to directly set a range of context-aware and event services so that it could provide 
context-aware serviced such as automatic control of the greenhouse environment, etc. and the SMS notification service for dangerous situations.

Figure 16. u-Greenhouse integrated management system GUI.

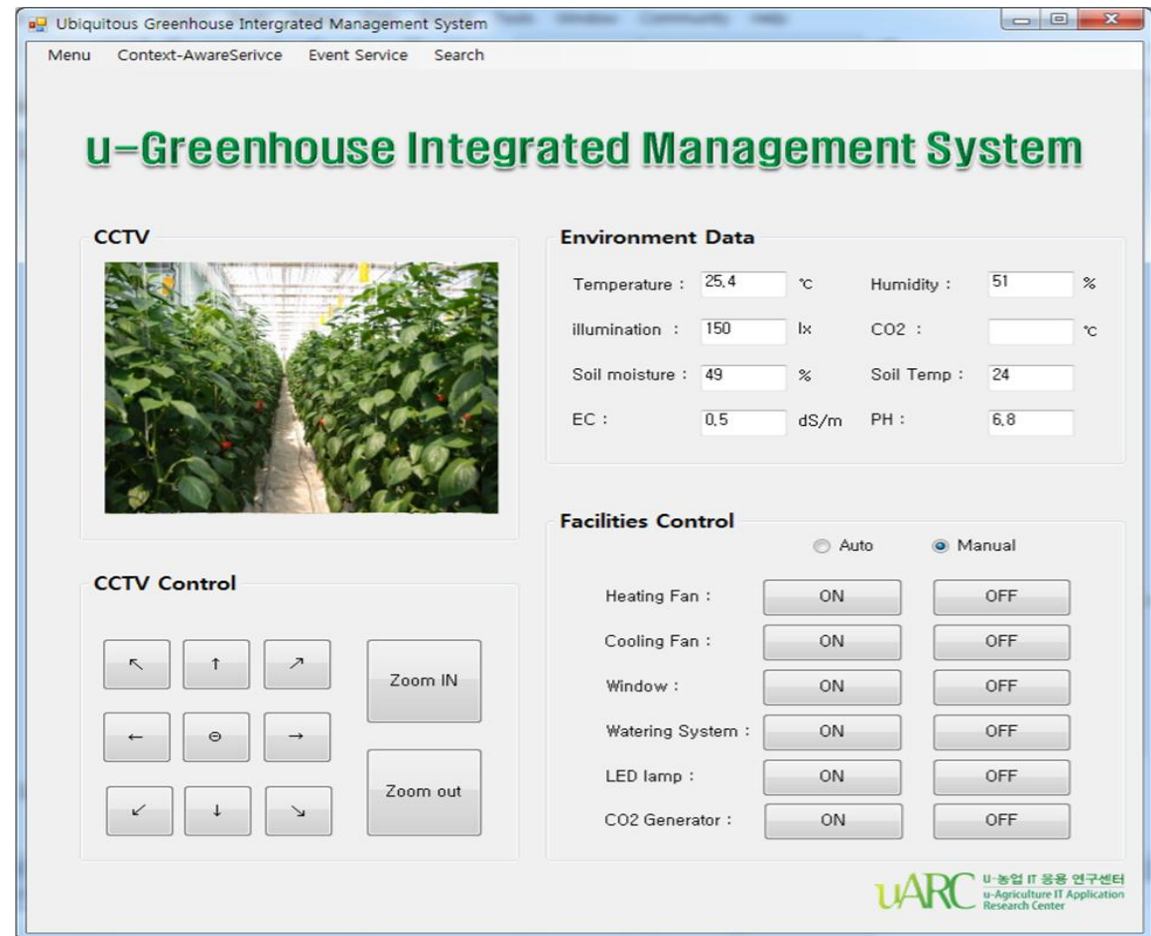

Figures 17 and 18 are screens that set a range of context-aware and event services.

Figure 17. Context-aware service GUI.

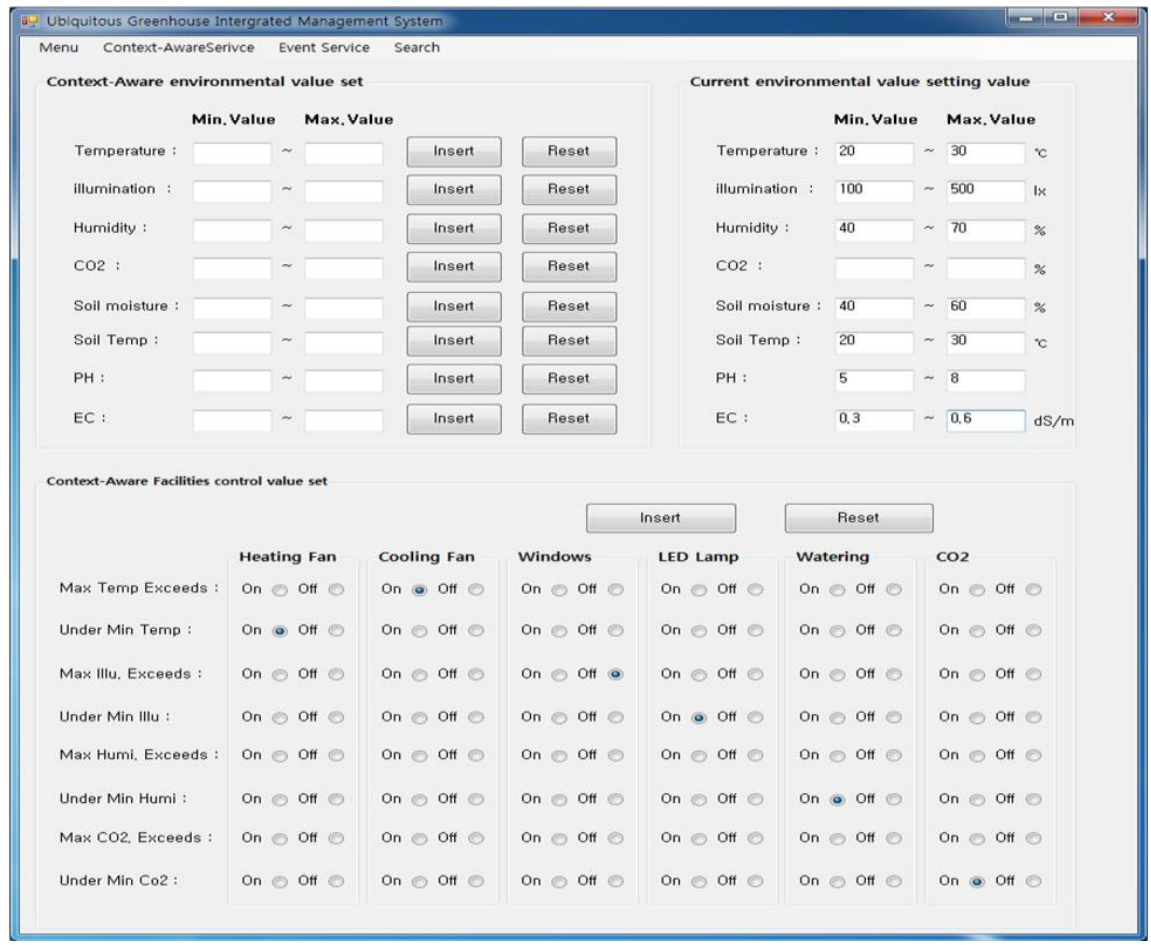


Figure 18. Event service GUI.

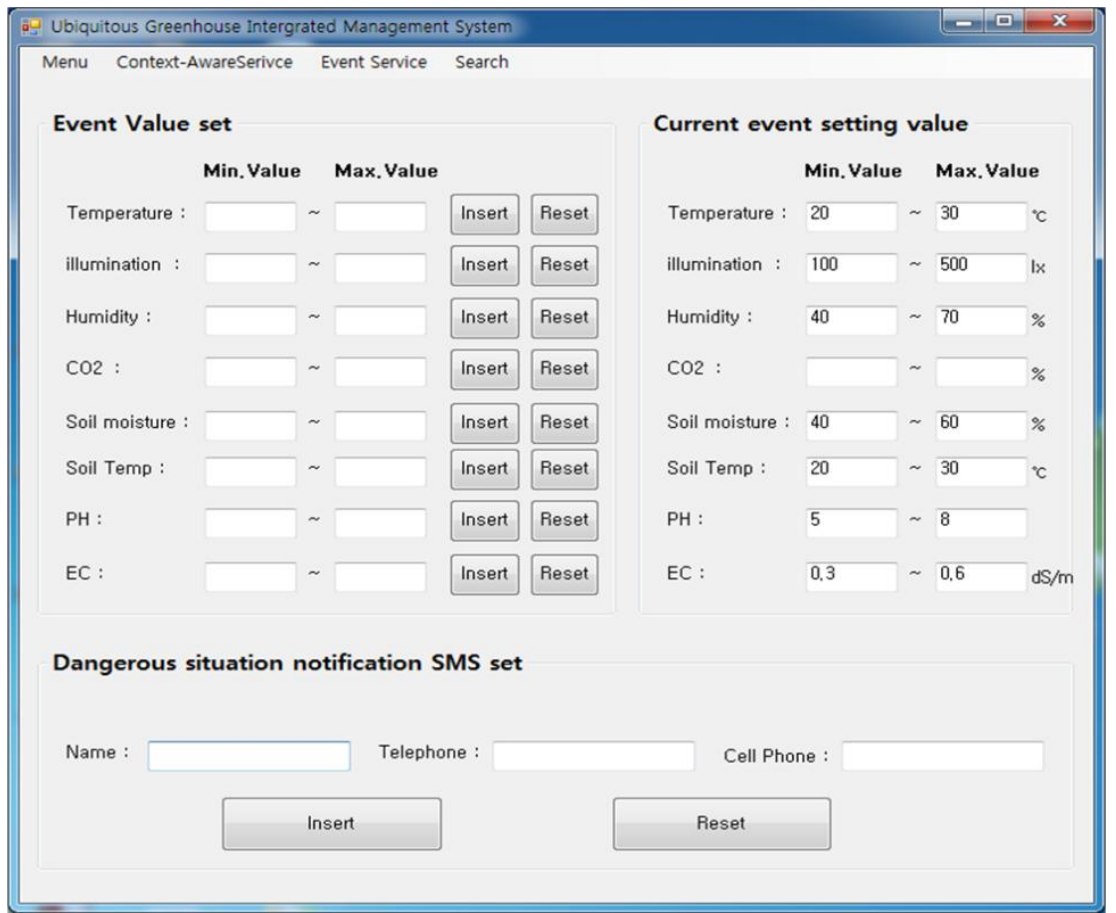

\subsection{Performance Evaluation of the Proposed Middleware}

This paper confirmed the proposed middleware's operability by measuring the server's load level and the response time for user's queries. To indirectly compare and measure the load level when integrating and processing data generated from lots of sensors, we used an application offered by Microsoft Windows. It is shown that CPU usage was about $20 \sim 30 \%$ on average when processing sensing data without middleware, and about $30 \sim 40 \%$ on average when processing data with the proposed middleware, as seen in Figure 19. Even though the value using the middleware is a little higher than the existing method processing data without middleware, it does not exceed $50 \%$ on average and considering that context-aware service, which it is not provided in the existing system, is added, therefore it could be confirmed that it is useful in terms of system usability.

Figure 19. Usage of CPU.

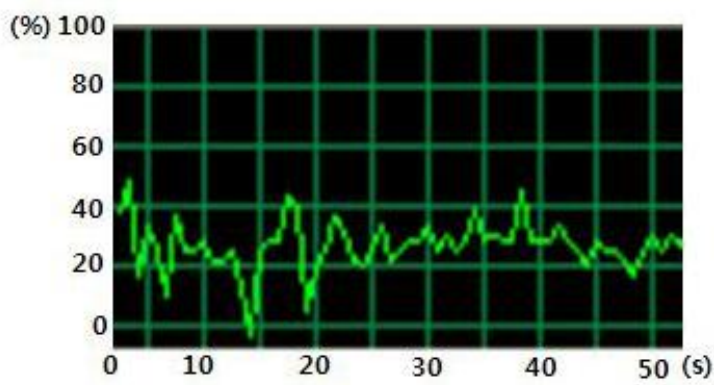

(a) Before applying Proposed middleware

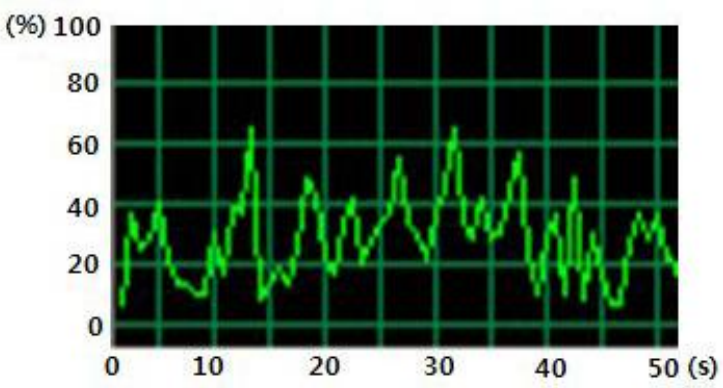

(b) After applying Proposed middleware

In addition, in order to measure average response time for user's requests the time required to obtain requested data from the server was simulated 50 times, and the result is as seen in Figure 20. 
Figure 20. Average response time for user's requests.

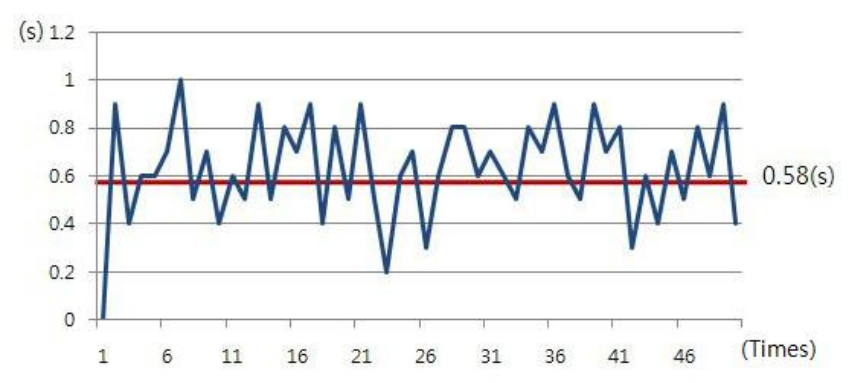

(a) Before applying Proposed middleware

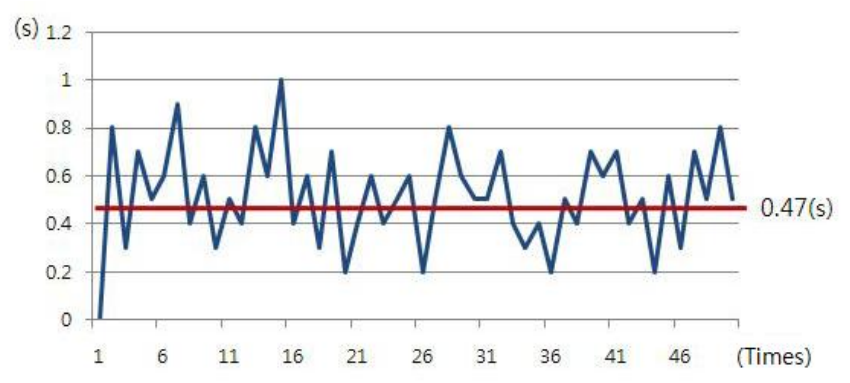

(b) After applying Proposed middleware

As seen in the figure, the response speed for user's requests was $0.58 \mathrm{~s}$ on average before using the middleware, and after applying the proposed middleware the average response speed was $0.47 \mathrm{~s}$, therefore, it could be seen that there was an improvement in terms of response speed.

\section{Conclusions}

This paper proposed and developed a context-aware middleware to efficiently process data collected from a u-Greenhouse applying WSN technologies and to implement combined services through organic connections of data. In order to solve the problem of dependence on certain hardware and operating systeme, which is a disadvantage of the existing middleware products, a novel middleware integrating different forms of data was suggested, and operability and scalability of the middleware was maximized by adding intelligent context-aware, event service, and filtering functions. In addition, to evaluate the performance of the proposed middleware, it was applied to an existing $\mathrm{u}$-Greenhouse to implement a u-Greenhouse integrated management system, and it could be confirmed that it was useful in terms of usability by comparing the CPU usage load level with that of the existing system and the response time for user's requests when operating the system. In future studies, the integrated middleware supporting abstraction between heterogeneous middleware for system's scalability by applying the future Web service should be studied, and expansion of ontology is needed for more accurate intelligent services. If such studies are reflected in the future versions of the middleware, it is expected that utilization of middleware would be increased.

\section{Acknowledgements}

This research was supported by the MKE(The Ministry of Knowledge Economy), Korea, under the ITRC(Information Technology Research Center) support program supervised by the NIPA(National IT Industry Promotion Agency)" (NIPA-2011-(C1090-1121-0009)).

\section{References}

1. Pyo, C.-S.; Chea, J.-S. Next-Generation RFID/USN Technology Development Prospects. Korea Inf. Commun. Soc. 2007, 24, 7-13.

2. Lee, M.-H.; Shin, C.-S.; Jo, Y.-Y.; Yoe, H. Implementation of Green House Integrated Management System in Ubiquitous Agricultural Environments. J. KIISE 2009, 27, 21-26. 
3. Shin, Y.-S. A Study on Informatization Model for Agriculture in Ubiquitous Era; MKE Research Report; National IT Industry Promotion Agency: Seoul, Korea, 2006.

4. Jeong, B.-M. Foreign u-Farm Service Model Casebook; NCA V-RER-06005; Issues and Analysis Report of Korea National Information Society Agency: Seoul, Korea, October 2006.

5. Kwon, O.-B.; Kim, J.-H. A Basic Direction for Building Agricultural Radio Frequency Identification Logistics Information System; Korea Rural Economics Institute: Seoul, Korea, December 2007.

6. Brandt Tracks Its Beef. Available online: http://www.rfidjournal.com/article/articleview/2229/1/1/ (accessed on 6 December 2010).

7. Kim, M.S.; Lee, Y.J.; Park, J.H. Trends of USN Middleware Technology; ETRI Electronic Communications Trend Report; Electronics and Telecommunications Research Institute: Daejeon, Korea, June 2007; Volume 22, pp. 67-79.

8. Hwang, J.H.; Yoe, H. Study of the Ubiquitous Hog Farm System Using Wireless Sensor Networks for Environmental Monitoring and Facilities Control. Sensors 2010, 10, 10752-10777

9. Hwang, J.H.; Shin, C.S.; Yoe, H. Study on an Agricultural Environment Monitoring Server System using Wireless Sensor Networks. Sensors 2010, 10, 11189-11211

10. Kim, Y.-M.; Han, J.I. Middleware Technology for Ubiquitous Sensor Network. J. KIISE 2007, 25, $35-48$.

11. Lee, K.W.; Kwon, H.E. Middleware Platform Technology for USN Environment. In Proceeding of 2008 Spring Conference Korean Institute of Intelligent Systems, Chungju, Korea, April 2008; Volume 18, pp. 85-86.

12. Hwang, J.G.; Cheong, T.S.; Kim, Y.I.; Lee, Y.J. Trends of RFID Middleware Technology and Its Applications; ETRI Electronic Communications Trend Report; Electronics and Telecommunications Research Institute: Daejeon, Korea, June 2005; Volume 20, pp. 81-91.

13. Xiong, N.; Vasilakos, A.V.; Yang, L.T.; Song, L.; Pan, Y.; Kannan, R.; Li, Y. Comparative Analysis of Quality of Service and Memory Usage for Adaptive Failure Detectors in Healthcare Systems. IEEE J. Sel. Areas Commun. 2009, 27, 495-509.

14. Xiong, N.; Jia, X.; Yang, L.T.; Vasilakos, A.V.; Pan, Y.; Li, Y. A Distributed Efficient Flow Control Scheme for Multi-Rate Multicast Networks. IEEE Trans. Parall. Distrib. Syst. 2010, 21, 1254-1266.

15. Zhou, L.; Xiong, N.; Shu, L.; Vasilakos, A.; Yeo, S.-S. Context-Aware Multimedia Service in Heterogeneous Networks. IEEE Intell. Syst. 2010, 25, 40-47.

16. Zhou, L.; Wang, X.; Tu, W.; Mutean, G.; Geller, B. Distributed Scheduling Scheme for Video Streaming over Multi-Channel Multi-Radio Multi-Hop Wireless Networks. IEEE J. Sel. Areas Commun. 2010, 28, 409-419.

17. Kung, S.H. The Design of Fungus Cultivating System based on USN. J. Korean Inst. Inf. Technol. 2007, 5, 34-41.

18. Levis, P.; Culler, D. Mate: A Virtual Machine for Tiny Networked Sensors. In Proceedings of ACM Conf. Architectural Support for Programming Languages and Operating Systems, San Jose, CA, USA, October 2002. 
19. Liu, T.; Martonosi, M. Impala: A Middleware System for Managing Autonomic, Parallel Sensor Systems. In Proceedings of ACM SIGPLAN Symposium on Principles and Practice of Parallel Programming, New York, NY, USA, June 2003; pp. 107-118.

20. Fok, C.-L.; Roman, G.-C.; Lu, C.Y. Mobile Agent Middleware for Sensor Networks: An Application Case Study. In Proceedings of the 4th International Conference on Information Processing in Sensor Networks (IPSN’05), Los Angeles, CA, USA, April 2005; pp. 382-387,

21. Yao, Y.; Gehrke, J. The Cougar Approach to In-Network Query Processing in Sensor Networks. SIGMOD Record 2002, 31, 9-18.

22. Shen, C.; Srisathapornphat, C.; Jaikeo, C. Sensor Information Networking Architecture and Applications. IEEE Personal Commun. 2001, 8, 52-59.

23. Heinzelman, W.B.; Murphy, A.L.; Carvalho, H.S.; Perillo, M.A. Middleware to Support Sensor Network Applications. IEEE Netw. 2004, 18, 6-14,

24. Kim, M.; Lee, J.W.; Lee, Y.J.; Ryou, J.C. COSMOS: A Middleware for Integrated Data Processing over Heterogeneous Sensor Networks. ETRI J. 2008, 30, 696-706.

25. Li, S.; Son, S.; Stankovic, J. Event Detection Services Using Data Service Middleware in Distributed Sensor Networks. In Proceedings of International Workshop on Information Processing in Sensor Networks (IPSN’03), Palo Alto, CA, USA, April 2003.

26. Madden, S.R.; Franklin, M.J.; Hellerstein, J.M. TinyDB: An Acquisitional Query Processing System for Sensor Networks. ACM TODS 2005, 30, 122-173.

27. Hwang, J.H.; Shin, C.S.; Yoe, H. A Wireless Sensor Network-Based Ubiquitous Paprika Growth Management System. Sensors 2010, 10, 11566-11589

28. Hanback ZigbeX II. Available online: http://www.hanback.co.kr/products/view/96 (accessed on 16 March 2011).

29. Park, D.-H.; Kang, B.-J.; Cho, K.-R.; Shin, C.-S.; Cho, S.-E.; Park, J.-W.; Yang, W.-M. A Study on Greenhouse Automatic Control System Based on Wireless Sensor Network. Wireless Pers. Commun. 2009, doi: 10.1007/s11277-009-9881-2.

30. MSP430 Mixed Signal Microcontroller. Available online: http://www.alldatasheet.com (accessed on 16 March 2011).

31. CC2420 2.4 GHz IEEE 802.15.4/Zigbee RF Transceiver. Available online: http://www.alldatasheet.com (accessed on 16 March 2011).

32. SHT71 Humidity \& Temperature Sensor. Available online: http://www.alldatasheet.com (accessed on 16 March 2011).

33. Protégé. Available online: http://protege.stanford.edu (accessed on 16 March 2011).

34. OWL. Available online: http://www.w3.org/TR/owl-features (accessed on 16 March 2011).

35. JENA, A Semantic Web Framework for Java. Available online: http://jena.sourceforge.net (accessed on 16 March 2011).

(C) 2011 by the authors; licensee MDPI, Basel, Switzerland. This article is an open access article distributed under the terms and conditions of the Creative Commons Attribution license (http://creativecommons.org/licenses/by/3.0/). 